\title{
Experienced Demand Does Not Affect Subsequent Sleep and the Cortisol Awakening Response
}

\section{Greg J Elder' \\ Mark A Wetherell ${ }^{2}$ \\ Thomas $\mathrm{V}$ Pollet $^{2}$ \\ Nicola L Barclay ${ }^{3}$ \\ Jason G Ellis $\mathbb{D}^{\prime}$}

'Northumbria Sleep Research Laboratory, Northumbria University, Newcastle Upon Tyne, UK; ${ }^{2}$ Department of Psychology, Northumbria University, Newcastle Upon Tyne, UK; ${ }^{3}$ Sleep and Circadian Neuroscience Institute, Nuffield Department of Clinical Neurosciences, University of Oxford, Oxford, UK
Correspondence: Greg J Elder

Northumbria Sleep Research Laboratory, Northumbria University, Newcastle Upon Tyne NEI 8ST, UK

Tel +44 I9l 227324 l

Email g.elder@northumbria.ac.uk
This article was published in the following Dove Press journal: Nature and Science of Sleep

Purpose: Stress is associated with subjective and objective sleep disturbances; however, it is not known whether stress disrupts sleep and relevant physiological markers of stress immediately after it is experienced. The present study examined whether demand, in the form of cognitive tasks, disrupted sleep and the cortisol awakening response (CAR), depending on whether it was experienced or just anticipated.

Participants and Methods: Subjective and objective sleep was measured in 22 healthy adults on three nights (Nights $0-2$ ) in a sleep laboratory using sleep diaries and polysomnography. Saliva samples were obtained at awakening, $+15,+30,+45$ and +60 minutes on each subsequent day (Day 1-3) and CAR measurement indices were derived: awakening cortisol levels, the mean increase in cortisol levels $(\mathrm{MnInc})$ and total cortisol secretion $\left(\mathrm{AUC}_{\mathrm{G}}\right)$. On Night 1, participants were informed that they were required to complete a series of demanding cognitive tasks within the sleep laboratory during the following day. Participants completed the tasks as expected or unexpectedly performed sedentary activities.

Results: Compared to the no-demand group, the demand group displayed significantly higher levels of state anxiety immediately completing the first task. There were no subsequent differences between the demand and no-demand groups in Night 2 subjective sleep continuity, objective sleep continuity or architecture, or on any Day 3 CAR measure.

Conclusion: These results indicate that sleep and the CAR are not differentially affected depending on whether or not an anticipated stressor is then experienced. This provides further evidence to indicate that the CAR is a marker of anticipation and not recovery. In order to disrupt sleep, a stressor may need to be personally relevant or of a prolonged duration or intensity.

Keywords: stress, cortisol, polysomnography, sleep, anticipation

\section{Introduction}

Stress has long been associated with disturbances to both subjective and objective sleep, ${ }^{1,2}$ and naturalistic studies have indicated that the anticipation of upcoming stress can disrupt subjective and objective sleep. ${ }^{3,4}$ A recent laboratory study demonstrated that anticipated stress, in the form of next-day demand, did not affect subjective or objective sleep. ${ }^{5}$ However, it is not known if stress can cause a "rebound" effect, whereby sleep is disrupted immediately (i.e. during the subsequent night) after the anticipated stressful event is then experienced.

Cortisol, which is the end product of the hypothalamic-pituitary-adrenal (HPA) axis, is responsive to psychological demand in a dose-response manner ${ }^{6}$ and is a 
suitable physiological marker of stress. The cortisol awakening response (CAR), which refers to the sharp increase in cortisol levels (of between approximately 38-75\%) which are observed in response to awakening, has been shown to be sensitive to anticipated demand. ${ }^{5,7}$ However, the CAR may also function as a marker of recovery from previous demand ${ }^{8}$ and is therefore an suitable physiological marker of assessing whether experiencing an anticipated stressor can disrupt the HPA axis.

The aim of the present study was to investigate whether an anticipated stressor subsequently disrupted subjective and objective sleep, and the CAR. Following the anticipation of a demanding day, it was expected that those who experienced demand would demonstrate poorer subjective and objective sleep, and an altered CAR profile, compared to those who did not.

\section{Participants and Methods}

\section{Participants}

Twenty-two healthy participants $\left(\mathrm{M}_{\mathrm{age}}=23.42\right.$ years; $\mathrm{SD}_{\text {age }}=3.62$ years, $50 \%$ male, $50 \%$ female) were recruited from the staff and student population of Northumbria University. Participants provided written informed consent and were paid $£ 150$ upon completion of the study.

Full screening procedures and detailed demographic information are reported in detail elsewhere. ${ }^{5}$ Briefly, participants were screened for current or previous sleep problems, physical or psychiatric illnesses, shift work or trans-meridian travel in the three months prior to study enrolment, using a structured clinical interview with a member of the sleep laboratory staff. Participants were not permitted to take part if there was any evidence of sleep difficulties or of current or previous physical/psychiatric illness. In addition, participants completed selfreported sleep diaries and two weeks of actigraphy prior to attending the sleep laboratory for the overnight part of the study. These were visually inspected by sleep laboratory staff in order to verify that sleep/wake schedules were stable prior to participation.

After consenting, participants were allocated to a demand $(\mathrm{n}=11)$ or no-demand $(\mathrm{n}=11)$ group. The demand group was intentionally recruited and completed the study before the no-demand group. This was a deliberate decision in order to ensure that the demand group did not reveal the true purpose of the study to the no-demand group in advance, due to the study population.

\section{Procedure}

The procedure has been previously described in detail elsewhere $^{5,9}$ and is summarised in Figure 1. Participants provided informed consent and were confirmed as being a healthy good sleeper by assessing their sleep, psychiatric

\section{Day -14 to Day 0}

- Completion of consent form

- Participants informed of demanding cognitive tasks (anticipation group only)

- Actigraphy

- Daily completion of sleep diaries

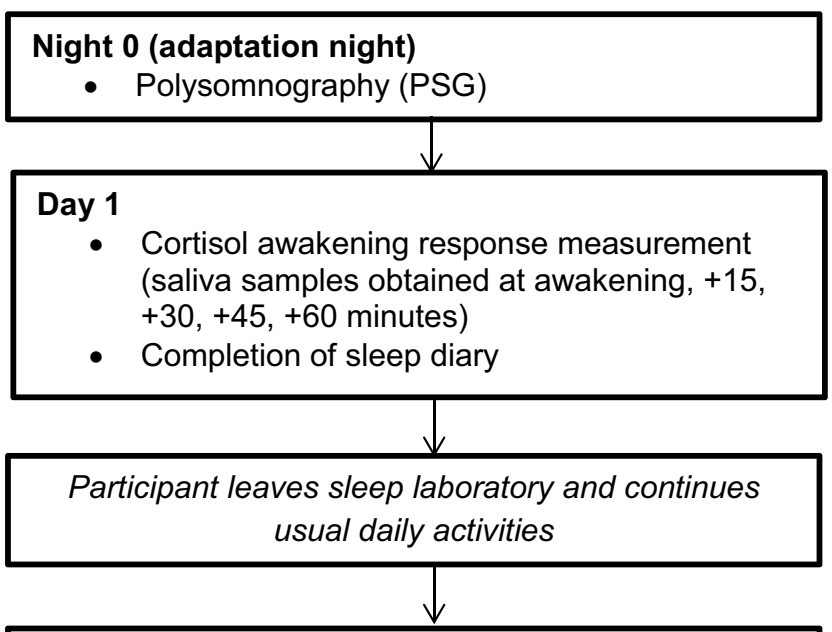

Night 1

- Participants informed of demanding cognitive tasks during Day 2 (anticipation group only)

- Polysomnography (PSG)

Day 2

- Cortisol awakening response measurement

- Completion of sleep diary

- Completion of cognitive tasks (demand group)

- Sedentary activities (no-demand group)

- Hourly state anxiety measurements (demand and no-demand groups)

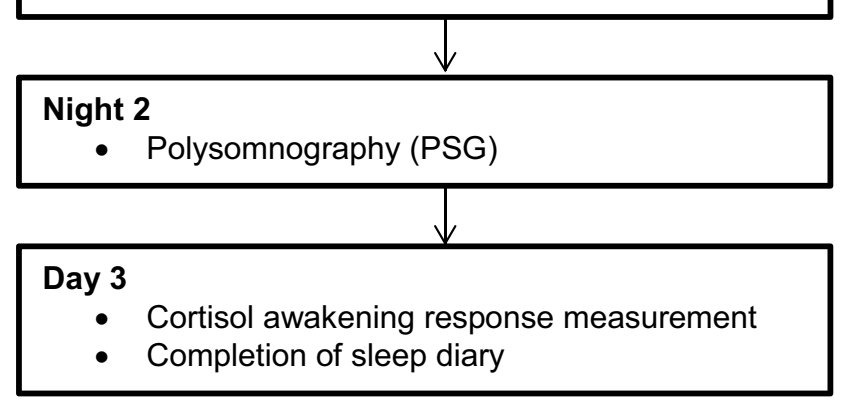

Figure I Study schematic. 
and physical illness history as described above. Participants also completed the Pittsburgh Sleep Quality Index $\left(\mathrm{PSQI}^{10}\right)$ and Hospital Anxiety and Depression Scale $\left(\mathrm{HADS}^{11}\right)$ as a measure of sleep quality, and of subjective anxiety and depression.

Participants completed a baseline period where sleep was monitored using sleep diaries and actigraphy (Days -14 to 0 ) before sleeping for three consecutive weekday nights in a sleep laboratory (Nights $0-2$ ). Lights out and wake-up times were scheduled in accordance with habitual (baseline sleep diary) times. Participants left the laboratory on Day 1, returned on Night 1, and remained under observation in the sleep laboratory until Day 3.

Sleep diaries ${ }^{12}$ were used to measure subjective sleep continuity (total sleep time (TST), time in bed (TIB), sleep efficiency $(\mathrm{SE} \%$ : $(\mathrm{TST} / \mathrm{TIB} \times 100))$, sleep-onset latency (SOL), number of awakenings (NWAK) and wake after sleep onset (WASO)) and polysomnography (PSG) was used to measure objective sleep. Mastoid and groundlinked EEG electrodes were placed at $\mathrm{FP}_{1}, \mathrm{FP}_{2}, \mathrm{~F}_{3}, \mathrm{~F}_{4}$, $\mathrm{C}_{3}, \mathrm{C}_{4}, \mathrm{P}_{3}, \mathrm{P}_{4}, \mathrm{O}_{1}, \mathrm{O}_{2}$ and $\mathrm{C}_{\mathrm{z}}$ and recordings were externally blind-scored in accordance with standard guidelines.13,14 For measurement of the CAR, saliva samples were collected at awakening, $+15,+30,+45$ and +60 minutes on three consecutive mornings (Day 1 - Day 3) using Salivettes (Sardstedt, Leicester, UK).

On Night 1, all participants were informed that they would remain in the sleep laboratory during Day 2 in order to complete a range of demanding cognitive tasks, where the best performance on a randomly-chosen task would be rewarded with a prize in order to elicit competition and arousal. On Day 2 from wake $+3 \mathrm{hrs}$ to $+13 \mathrm{hrs}$, the participants in the demand condition $(n=11)$ completed hourly computerised tasks of 10-15 minutes in duration (Emotional Stroop task, ${ }^{15}$ Multi-Tasking Framework ${ }^{16}$ and Iowa Gambling Task ${ }^{17}$ ). As a measure of state anxiety, all participants responded to statements from the shortform state anxiety scale, ${ }^{18}$ using $100 \mathrm{~mm}$ visual analogue scales, where $0 \mathrm{~mm}$ indicated "not at all" and $100 \mathrm{~mm}$ indicated "very much". State anxiety was measured at wake +60 minutes, and hourly thereafter, except at meal breaks (provided at wake $+2 \mathrm{hrs}$, $+6 \mathrm{hrs}$ and $+10 \mathrm{hrs}$ ).

On Day 2 the no-demand group $(n=11)$ was informed that they were not required to complete any tasks, and instead remained in the sleep laboratory performing sedentary activities including reading and watching television.

\section{Data Analysis}

Night 2 subjective and objective sleep, and Day 3 CAR data are reported. Measures of subjective sleep continuity (TIB, TST, SE\%, SOL, NWAK and WASO), objective sleep continuity (TST, SE\%, SOL, NWAK and WASO) and objective sleep architecture (percentages of sleep spent in REM, N1, N2 and N3) were compared between groups using $t$-tests adjusted for multiple comparisons (adjusted $p$-values $=0.008,0.013$ and 0.006).

CAR data from five participants (demand $\mathrm{n}=2$; no demand $n=3$ ) were excluded due to saliva samples containing an insufficient volume of saliva for analysis. The CAR was examined by comparing cortisol levels (nanomoles per litre; nmol/l) between groups using a $2 \times 5$ mixed analysis of variance (ANOVA). Additional CAR indices were compared between groups using $t$-tests: awakening cortisol levels, the mean increase in cortisol levels during the measurement period $\left(\mathrm{MnInc}^{19}\right)$ and total cortisol secretion, expressed as the area under the curve with respect to ground $\left(\mathrm{AUC}_{\mathrm{G}}\right)$, adjusted for multiple comparisons (adjusted $p$-value $=$ 0.017). Effect sizes are reported using Cohen's $d$. State anxiety was compared between groups using a $2 \times 10$ mixed ANOVA, with follow-ups adjusted for multiple comparisons (adjusted $p$-value $=0.005$ ).

Table I Night 2 Subjective Sleep Continuity Comparisons

\begin{tabular}{|l|l|l|l|l|l|l|}
\hline \multirow{2}{*}{} & \multicolumn{2}{|l|}{ Demand $(\mathbf{n}=\mathbf{I}$ ) } & \multicolumn{2}{l|}{ No Demand $(\mathbf{n}=\mathbf{I})$} & \multirow{2}{*}{ p-value } & \multirow{2}{*}{ Effect Size (d) } \\
\cline { 2 - 6 } & Mean & SD & Mean & SD & \\
\hline TIB (mins) & 532.27 & 42.80 & 539.55 & 44.52 & 0.700 & 0.17 \\
TST (mins) & 455.82 & 60.45 & 470.27 & 40.04 & 0.516 & 0.30 \\
SOL (mins) & 21.36 & 16.45 & 10.68 & 5.25 & 0.063 & 0.92 \\
NWAK & 1.00 & 0.89 & 1.18 & 1.15 & 0.683 & 0.18 \\
WASO (mins) & 5.82 & 6.66 & 3.36 & 5.84 & 0.369 & 0.41 \\
SE (\%) & 85.44 & 7.70 & 87.20 & 3.27 & 0.495 & 0.31 \\
\hline
\end{tabular}

Abbreviations: TIB, time in bed; TST, total sleep time; SOL, sleep onset latency; NWAK, number of awakenings; WASO, wake after sleep onset; SE, sleep efficiency. 
Table 2 Night 2 Demand and No-Demand Group Objective Sleep Comparisons

\begin{tabular}{|c|c|c|c|c|c|c|}
\hline & \multicolumn{2}{|c|}{ Demand $(n=I I)$} & \multicolumn{2}{|c|}{ No Demand $(n=I I)$} & \multirow[t]{2}{*}{ p-value } & \multirow[t]{2}{*}{ Effect Size (d) } \\
\hline & Mean & $S D$ & Mean & $S D$ & & \\
\hline TST (mins) & 443.00 & 39.48 & 456.86 & 40.48 & 0.426 & 0.36 \\
\hline SOL (mins) & 13.23 & 10.25 & 8.27 & 5.40 & 0.176 & 0.64 \\
\hline NWAK & 12.73 & 4.34 & 10.91 & 4.44 & 0.343 & 0.43 \\
\hline WASO (mins) & 12.45 & 10.22 & 9.55 & 9.17 & 0.490 & 0.31 \\
\hline SE (\%) & 94.55 & 2.74 & 96.21 & 2.13 & 0.128 & 0.71 \\
\hline Time in REM (\%) & 23.86 & 6.32 & 24.08 & 7.33 & $0.94 I$ & 0.03 \\
\hline Time in NI (\%) & 3.74 & 1.76 & 2.62 & 1.46 & 0.121 & 0.73 \\
\hline Time in N2 (\%) & 51.48 & 9.13 & 51.19 & 7.03 & 0.934 & 0.04 \\
\hline Time in N3 (\%) & 20.90 & 6.66 & 22.10 & 5.67 & 0.654 & 0.20 \\
\hline Latency to REM (mins) & 102.27 & 57.93 & 95.00 & 46.38 & 0.749 & 0.15 \\
\hline Latency to NI (mins) & 13.23 & 10.25 & 8.27 & 5.40 & 0.176 & 0.64 \\
\hline Latency to N2 (mins) & 21.14 & 9.88 & 13.68 & 7.24 & 0.057 & 0.90 \\
\hline Latency to N3 (mins) & 33.95 & 14.00 & 26.23 & 9.96 & 0.151 & 0.67 \\
\hline
\end{tabular}

Abbreviations: TST, total sleep time; SOL, sleep onset latency; NWAK, number of awakenings; WASO, wake after sleep onset; SE, sleep efficiency; REM, rapid eye movement sleep; NI, stage I sleep; N2, stage 2 sleep; N3, stage 3 sleep.

\section{Results}

Participant sleep quality was within the normal range (PSQI $M=3.36, S D=1.89$ ), as was subjective anxiety (HADS Anxiety $M=5.41, S D=3.17$ ) and subjective depression (HADS Depression sub-scale $M=2.36, S D=1.97$ ).

There were no between-group differences in any subjective or objective measure of sleep continuity or architecture ( $p$-values $>0.05$; Tables 1 and 2). Cortisol levels showed a main effect of time point $(F(2.52,37.74)=0.94, p<0.001$, $\eta 2_{\mathrm{p}}=0.06$; Figure 2), representing a typical increase in cortisol levels during the CAR measurement period. The time point $\times$ group interaction, and main effect of group, was not significant ( $p$-values $>0.05)$. There were no significant between-group differences in awakening cortisol levels, MnInc, or total cortisol secretion ( $p$-values $>0.05$; Table 3 ).

For state anxiety, the main effect of time point was not significant $(p>0.05)$. There was a significant main effect of condition $\left(F(1,19)=6.68, p=0.018, \eta 2_{\mathrm{p}}=0.26\right.$, and $\mathrm{a}$ significant time point $\times$ group interaction $(F(4.06,77.17)=$ $2.51, p=0.048, \eta 2_{\mathrm{p}}=0.12$. Follow-up comparisons indicated

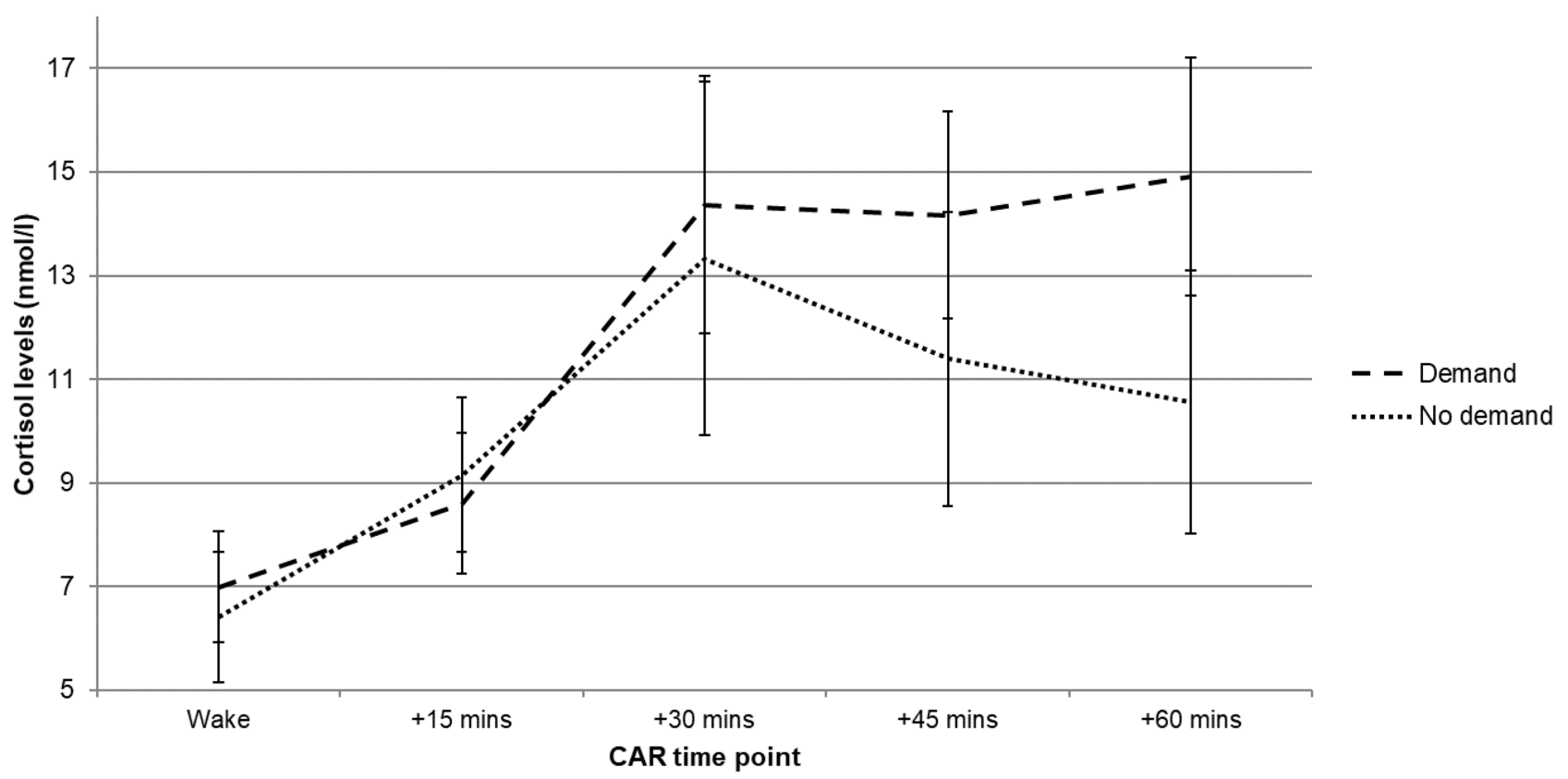

Figure 2 Day 3 mean $( \pm$ SEM) CAR profile comparisons between demand and no-demand groups. There were no significant differences $(p$-values $>0.05)$. 
Table 3 Day 3 Additional Cortisol Awakening Response Measurement Indices by Group

\begin{tabular}{|l|l|l|l|l|l|l|}
\hline \multirow{2}{*}{} & \multicolumn{2}{|l|}{ Demand (n = 9) } & \multicolumn{2}{l|}{ No Demand (n=8) } & \multirow{2}{*}{ p-value } & \multirow{2}{*}{ Effect Size (d) } \\
\cline { 2 - 5 } & Mean & SD & Mean & SD & & \\
\hline Awakening levels (nmol/l) & 7.00 & 3.79 & 6.41 & 3.04 & 0.731 & 0.18 \\
AUC $(\mathrm{nmol} / \mathrm{l})$ & 721.30 & $396.5 \mathrm{I}$ & 635.44 & 258.62 & 0.610 & 0.27 \\
Mnlnc (nmol/) (arbitrary units) & 6.02 & 5.58 & 4.70 & 3.43 & 0.574 & 0.30 \\
\hline
\end{tabular}

Abbreviations: $\mathrm{AUC}_{\mathrm{G}}$, area under the curve with respect to ground; Mnlnc, mean increase.

that the demand group showed significantly higher levels of state anxiety at wake $+3 \mathrm{hrs}(p=0.003)$, occurring immediately after the demand group completed the first task (Figure 3).

\section{Discussion}

This study investigated whether an anticipated stressor subsequently disrupted subjective sleep, objective sleep, and the CAR. There were no subsequent differences in subjective or objective sleep between participants who experienced demand and participants who did not experience demand. This indicates that an anticipated stressor does not cause a disruptive "rebound" effect upon sleep following the stressor. There were no statistically significant between-group differences in the CAR of the following day. There was, however, a trend towards increased cortisol levels from wake to +30 minutes in the demand group and this is likely to indicate that there are high levels of individual differences in the CAR following an anticipated stressor. In order to confirm whether or not this is the case with the CAR, these findings should be replicated and investigated further in a larger sample.

The demand placed upon participants occurred repeatedly at multiple time points throughout the day, was of a greater duration than the anticipated demand in previous stress-induction studies ${ }^{20}$ and demonstrably increased levels of state anxiety in comparison to participants who did not experience the demand. However, it is still possible that a stressor may need to be of a greater intensity, severity or duration, or that a stressor may need to be personally-relevant, in order to disrupt subjective or objective sleep. Alternatively, the advance warning of the demand may have allowed participants to prepare and therefore mitigate the impact upon sleep, as is suggested by the CAR results in the present study.

A particular strength of the study is in the highly-controlled sleep laboratory environment, which ensured complete control over relevant environmental factors including

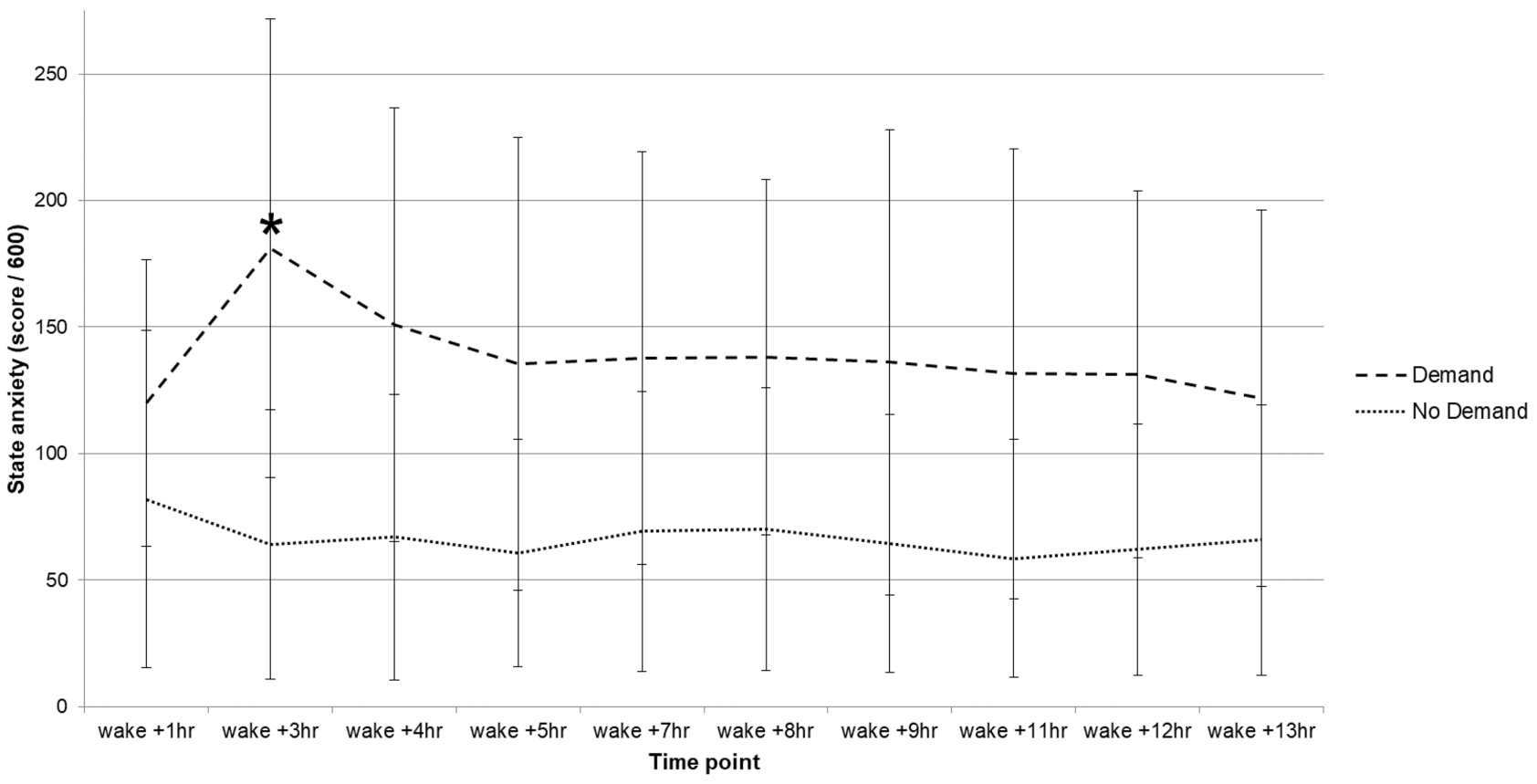

Figure 3 Day 3 state anxiety levels between demand and no-demand groups $\left({ }^{*} p<0.005\right)$. 
light levels, participant food intake and exercise, as well as ensuring accurate saliva sample collection. ${ }^{21}$ This is important since short delays to sample collection can lead to an inaccurate $\mathrm{CAR}^{22} \mathrm{~A}$ limitation is in the relatively small sample size, and although the high level of control offers advantages over ambulatory studies, these findings should still be considered to be preliminary and should be replicated with larger samples.

\section{Conclusions}

Overall, an anticipated stressor does not disrupt objective sleep in the subsequent night, or the CAR during the subsequent day; this indicates that the CAR is not a marker of recovery ${ }^{8}$ and is instead a marker of anticipation. ${ }^{5,7}$ In order to disrupt sleep, the stressor may need to be personally relevant, or of a longer duration or intensity.

\section{Abbreviations}

ANOVA, analysis of variance; $\mathrm{AUC}_{\mathrm{G}}$, area under the curve with respect to ground; CAR, cortisol awakening response; EEG, electroencephalography; EMG, electromyography; MnInc, mean increase; N1, non-rapid eye movement stage 1; N2, non-rapid eye movement stage 2; N3, non- rapid eye movement stage 3; NWAK, number of awakenings; PSG, polysomnography; REM, rapid eye movement; SE, sleep efficiency; SOL, sleep onset latency; TIB, time in bed; TST, total sleep time; WASO, wake after sleep onset.

\section{Ethics Approval and Informed Consent}

This study was ethically approved by the Northumbria University Faculty of Health and Life Sciences ethics committee. All participants provided written informed consent.

\section{Acknowledgments}

We would like to thank all study participants. We thank Dr. Zoe Gotts, Dr. Rachel Sharman, Dr. Umair Akram for their assistance with data collection and Anthea Wilde for conducting the saliva analyses. This study was financially supported by Northumbria University.

\section{Author Contributions}

GE led data analysis and wrote and drafted the manuscript. All authors (GE, MW, TP, NB and JE) contributed to the study design, data analysis and subsequent drafts of the manuscript. All authors contributed to data analysis, drafting and revising the article, gave final approval of the version to be published, and agree to be accountable for all aspects of the work.

\section{Funding}

This study was financially supported by Northumbria University.

\section{Disclosure}

The authors declare that they have no financial or nonfinancial competing interests.

\section{References}

1. Bastien CH, Vallieres A, Morin CM. Precipitating factors of insomnia. Behav Sleep Med. 2004;2(1):50-62.doi:10.1207/s15402010bsm0201_5

2. Reynolds III CF, Hoch CC, Buysse DJ, et al. Sleep after spousal bereavement: a study of recovery from stress. Biol Psychiatry. 1993;34(11):791-797. doi:10.1016/0006-3223(93)90068-O

3. Torsvall L, Akerstedt T. Disturbed sleep while being on-call: an EEG study of ships' engineers. Sleep. 1988;11(1):35-38. doi:10.1093/ sleep/11.1.35

4. Torsvall L, Castenfors K, Åkerstedt T, Fröberg JAN. Sleep at sea: a diary study of the effects of unattended machinery space watch duty. Ergonomics. 1987;30(9):1335-1340. doi:10.1080/00140138708966027

5. Elder GJ, Barclay NL, Wetherell MA, Ellis JG. Anticipated next-day demand affects the magnitude of the cortisol awakening response, but not subjective or objective sleep. J Sleep Res. 2018;27(1):47-55. doi: $10.1111 /$ jsr. 12569

6. Dickerson SS, Kemeny ME. Acute stressors and cortisol responses: a theoretical integration and synthesis of laboratory research. Psychol Bull. 2004;130(3):355-391. doi:10.1037/0033-2909.130.3.355

7. Wetherell MA, Lovell B, Smith MA. The effects of an anticipated challenge on diurnal cortisol secretion. Stress. 2015;18(1):42-48. doi:10.3109/10253890.2014.993967

8. Adam EK, Hawkley LC, Kudielka BM, Cacioppo JT. Day-to-day dynamics of experience-cortisol associations in a population-based sample of older adults. Proc Natl Acad Sci U S A. 2006;103 (45):17058-17063. doi:10.1073/pnas.0605053103

9. Elder GJ, Ellis JG, Barclay NL, Wetherell MA. Assessing the daily stability of the cortisol awakening response in a controlled environment. BMC Psychol. 2016;4(1):1-10. doi:10.1186/s40359-016-0107-6

10. Buysse DJ, Reynolds III CF, Monk TH, Berman SR, Kupfer DJ. The pittsburgh sleep quality index: a new instrument for psychiatric practice and research. Psychiatry Res. 1989;28(2):193-213. doi:10.1016/0165-1781(89)90047-4

11. Zigmond AS, Snaith RP. The hospital anxiety and depression scale. Acta Psychiatr Scand. 1983;67(6):361-370. doi:10.1111/j.16000447.1983.tb09716.x

12. Carney CE, Buysse DJ, Ancoli-Israel S, et al. The consensus sleep diary: standardizing prospective sleep self-monitoring. Sleep. 2012;35(2):287-302. doi:10.5665/sleep.1642

13. Iber C, Ancoli-Israel S, Quan SF. The AASM Manual for the Scoring of Sleep and Associated Events: Rules, Terminology and Technical Specifications. Westchester: American Academy of Sleep Medicine; 2007.

14. Iber C, Ancoli-Israel S, Quan SF. The AASM Manual for the Scoring of Sleep and Associated Events: Rules, Terminology and Technical Specifications (Version 2.6). Westchester: American Academy of Sleep Medicine; 2020. 
15. Barclay NL, Ellis JG. Sleep-related attentional bias in poor versus good sleepers is independent of affective valence. J Sleep Res. 2013;22(4):414-421. doi:10.1111/jsr.12035

16. Wetherell MA, Sidgreaves MC. Secretory immunoglobulin-A reactivity following increases in workload intensity using the Defined Intensity Stressor Simulation (DISS). Stress Health. 2005;21(2):99106. doi:10.1002/smi.1038

17. Bechara A, Damasio AR, Damasio H, Anderson SW. Insensitivity to future consequences following damage to human prefrontal cortex Cognition. 1994;50(1-3):7-15. doi:10.1016/0010-0277(94)90018-3

18. Marteau TM, Bekker H. The development of a six-item short-form of the state scale of the Spielberger State-Trait Anxiety Inventory (STAI). Br J Clin Psychol. 1992;31(3):301-306. doi:10.1111/j.20448260.1992.tb00997.x
19. Wüst S, Wolf J, Hellhammer DH, Federenko I, Schommer N, Kirschbaum C. The cortisol awakening response - normal values and confounds. Noise Health. 2000;2(7):79-88.

20. Hall M, Vasko R, Buysse D, et al. Acute stress affects heart rate variability during sleep. Psychosom Med. 2004;66(1):56-62. doi:10.1097/01.PSY.0000106884.58744.09

21. Elder GJ, Wetherell MA, Barclay NL, Ellis JG. The cortisol awakening response - applications and implications for sleep medicine. Sleep Med Rev. 2014;18(3):195-204. doi:10.1016/j.smrv.2013.05.001

22. Smyth N, Clow A, Thorn L, Hucklebridge F, Evans P. Delays of 5-15 min between awakening and the start of saliva sampling matter in assessment of the cortisol awakening response. Psychoneuroendocrinology. 2013;38(9):1476-1483. doi:10.1016/j. psyneuen.2012.12.013

\section{Publish your work in this journal}

Nature and Science of Sleep is an international, peer-reviewed, open access journal covering all aspects of sleep science and sleep medicine, including the neurophysiology and functions of sleep, the genetics of sleep, sleep and society, biological rhythms, dreaming, sleep disorders and therapy, and strategies to optimize healthy sleep.
The manuscript management system is completely online and includes a very quick and fair peer-review system, which is all easy to use. Visit http://www.dovepress.com/testimonials.php to read real quotes from published authors. 